\title{
Unavoidable trapped mode in the interaction region of colliding beams
}

\author{
Alexander Novokhatski* and Michael Sullivan \\ SLAC National Accelerator Laboratory, Menlo Park, California 94025, USA \\ Eleonora Belli, Miguel Gil Costa, and Roberto Kersevan \\ CERN, 1211 Geneva 23, Switzerland \\ (Received 2 August 2017; published 22 November 2017)
}

\begin{abstract}
We discuss the nature of the electromagnetic fields excited by the beams in the beam pipe of an interaction region. In trying to find an optimum geometry for this region with a minimum of electromagnetic wave excitation, we have discovered one mode, which remains even in a very smooth geometry. This mode has a longitudinal electrical component and can be easily excited by the beam. By analyzing the structure of this mode we have found a way to absorb this mode. The work was done in connection with a proposal for a future electron-positron collider.
\end{abstract}

DOI: 10.1103/PhysRevAccelBeams.20.111005

\section{INTRODUCTION}

One of the ways to reach a higher luminosity in colliders is to increase the beam currents. Higher beam currents produce more electromagnetic waves in the interaction region (IR) due to the diffraction of the beam fields from the inhomogeneous beam pipe. The structure of the electromagnetic fields excited in an IR is very complicated. Usually each of the colliding beams has a different incoming beam pipe. These beam pipes are combined into one pipe near the collision point. The beams do not generate electromagnetic fields in the smooth part of the beam chamber; however in the interaction region they may generate a lot of electromagnetic waves due to chamber irregularities. Some of the low frequency waves can be trapped in the IR. A trapped mode is characterized by its resonant frequency and the effective shunt impedance. It can happen that the mode resonant frequency may be equal to the frequency of some of the revolution harmonics. In this case, the amplitude of the electromagnetic field of this mode will grow until it reaches the value determined by the loaded quality factor together with the loss factor of this mode. The mode loss factor is determined by the interaction of a beam particle with the field of this mode. To interact with a beam particle, the trapped mode must have a longitudinal electrical component, which is collinear with the beam particle velocity. For this reason not all existing higher order modes (HOMs) will interact with a beam particle. Typically, the frequency of HOMs in the interaction region is in the range of several $\mathrm{GHz}$. If some of the

*novo@slac.stanford.edu

Published by the American Physical Society under the terms of the Creative Commons Attribution 4.0 International license. Further distribution of this work must maintain attribution to the author(s) and the published article's title, journal citation, and DOI.
HOMs have a large quality factor, of order more than 1000 , and the frequency of the mode is in resonance with some harmonic of the revolution frequency, then local heating in the IR can reach tens of $\mathrm{kW}$ of power. Other electromagnetic waves, excited by the beam, with a frequency above the cutoff frequency will travel away from the IR and go down the beam pipe. The absorption of these waves can bring heating problems to other parts of the accelerator. A large energy loss of the beams in the interaction region can be a severe problem. The temperature of the IR chamber will go up and the vacuum will be spoiled. If the IR chamber has small gaps or hidden cavities (like in shielded bellows or valves), then electric sparks or arcing may cause additional vacuum spikes. Heating of nonevaporable getters (if they are used in the IR) may bring vacuum instability (the temperature can go above the recovery level). All of these things can make the backgrounds much higher. One can find a description of these effects in publications [1,2].

Detuning (making the mode frequency not equal to any harmonic of the revolution frequency) and the suppression of the loaded quality factor seem to be the only ways to decrease the HOM heating power.

However during the design of the IR we can try to diminish these effects by making the geometry of the IR very smooth and consequently reduce the number of trapped HOMs. In analyzing this problem we found that one mode will still stay in the IR even when the geometry of the metal chamber is very smooth. Of course the reason for this is in the geometry of the connection of two tubes. We will present a description of this mode, which we obtained using two different methods of electromagnetic calculation. One method is by a planar geometry. This method was developed for coherent synchrotron radiation and wakefield simulations [3]. We use this method to make a quick estimate of the power loss, however for a more accurate estimate we did 3D calculations using CST [4] and HFSS [5] codes. 
We may note that wakefield calculations have been done for IRs of different colliders [6-14]. The IR geometry was mainly determined by the detector setup and shielding of the synchrotron radiation. However the predictions of energy loss in the IR in some publications are many times different and do not match experimental measurements [13-14]. In some publications [8] one can find a very small energy loss of order of ten watts, while another publication predicts [12] an enormous power loss of $1 \mathrm{MW}$ in the IR. It is very difficult to imagine that the interaction region can be a highly efficient cw klystron of megawatt power.

In this paper we describe a study focused on reducing the beam impedance of the interaction region beam pipe in the proposed Future Circular electron-positron Collider (FCC-ee) [15]. We first analyze the nature of the excitation of the fields in the interaction region using $2 \mathrm{D}$ and $3 \mathrm{D}$ codes for numerical simulations. The simulations led to the discovery of a trapped mode that seems to be always present in the IR beam pipes. We then carefully describe this mode and its field structure. This follows by studying three different beam pipe models proposed for the FCC-ee IR. A conclusion and an Appendix finalize the paper.

\section{WAVE EXCITATION IN THE IR}

We start by explaining the reason why we have an additional excitation of the electromagnetic fields in the IR. When a bunch is coming from one connecting pipe and passing the junction point where another pipe connects a new electromagnetic field appears in the other connected pipe. At the same time, some part of the bunch field returns back into the other connecting pipe. Figure 1 shows the electric field line distribution for a time when a bunch just passed the beam pipe connection. We can see that the bunch has extended its self-field into the larger common pipe, while some part of the field propagates back into the other

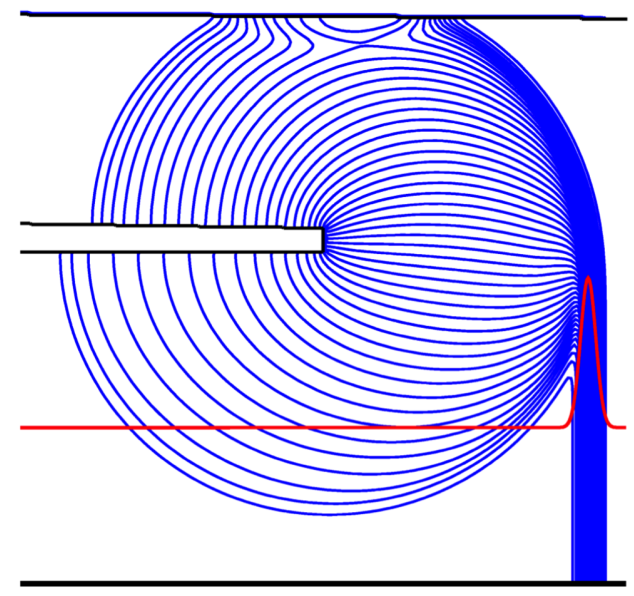

FIG. 1. Electric force field line distribution at the time when a relatively short bunch has just passed a pipe connection. The red line shows the bunch line charge density distribution and the bunch trajectory. pipe and some part is reflected back into the original beam pipe. Also we can see that an image charge appears at the edge of the pipe connection. This charge and later the image current (running across the pipes) produces electromagnetic fields in both pipes. This field propagates in time from the edge of the pipe connection. We need to note that a shorter bunch excites more fields in the pipes, due to the excitation of the higher frequency fields, which may freely propagate in the pipe.

In the common central pipe of the IR the electromagnetic fields, which were excited at the edge of the pipe connection chase the bunch. In time the bunch forms a transverse self-field and loses kinetic energy because of this. The self-field is also changing due to the bunch trajectory in the common pipe. The bunch is close to one side near the incoming pipe connection and then goes to the other side of the pipe in order to get into the opposite outgoing pipe. The bunch gets more deceleration as it excites freely propagating electromagnetic fields in this region.

After a bunch has passed the central common pipe it enters one of the outgoing pipes. Now a newly formed selffield is diffracted at the edge of the pipe connection. Mainly, it is cut by the edge and then propagates into both pipes. The plot of the electric force line distribution for the time when a bunch has passed the outgoing pipe connection is shown in Fig. 2. In this plot the green lines show force lines with different direction in comparison with other force lines that are opposite to the force lines shown in blue. Electromagnetic fields, excited by a short bunch are very complicated in this region, because there are a lot of fields, mainly high frequency waves, which are still chasing the bunch after the pipe connection at the entrance of the IR.

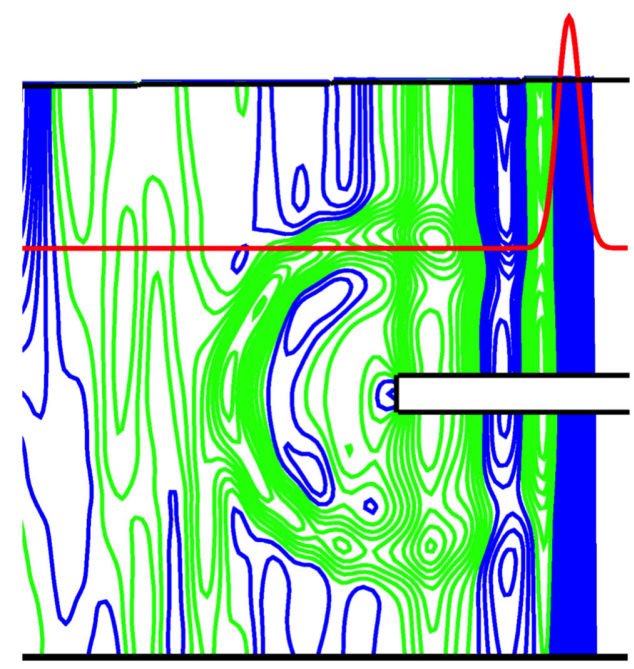

FIG. 2. Electromagnetic field line distribution at the time when a short bunch has passed the ougoing pipe connection. The green lines show force lines with an opposite direction in comparison with other force lines shown in blue. The red line shows the bunch line charge density distribution and the bunch trajectory. 
Nevertheless among these fields we can see the field from an image charge at the edge of the pipe connection in Fig. 2. Some part of the field is reflected back into the common central pipe. This field is the source of the HOMs, which will be formed after many reflections. Also, there is some part of the field, which continues to chase the bunch. We cannot avoid exciting these fields in the interaction region.

\section{STRUCTURE OF THE UNAVOIDABLE TRAPPED MODE IN THE IR}

In trying to find an optimal geometry for the IR with minimum electromagnetic wave resonance excitation (minimum impedance), we found that one mode remains even in a very smooth geometry. First calculations were done using a flat version of the code NOVO for a flat geometry in the IR. This mode is situated near the connection of the two incoming and outgoing pipes. The electric field distribution and the surface current are shown in Fig. 3 (corresponding left and right plot). The surface charges are concentrated at the edge of the pipe connection and the top and bottom sides of the common central pipe. The surface image currents appear at the time when the image charges start to move to each other. Then the image charges exchange each other. Another image current with opposite sign will change the sign of charges again and oscillations will continue. The frequency of this mode is slightly lower than the cutoff frequency of the common pipe due to the concentration of the electric fields at the edge of the pipe connection. Naturally the cutoff frequency of the incoming pipes is much higher than that of the mode frequency. We therefore have a real trapped mode.

This mode is a combination of a longitudinal and a transverse mode. It can be seen in Fig. 3 that this mode has a longitudinal electric component parallel to the bunch trajectories and can be easily excited by the colliding beams. A bunch produces a stronger interaction with this mode if it travels closer to the pipe connection. The transverse kick from this mode in the incoming pipe connection will be compensated in the outgoing pipe

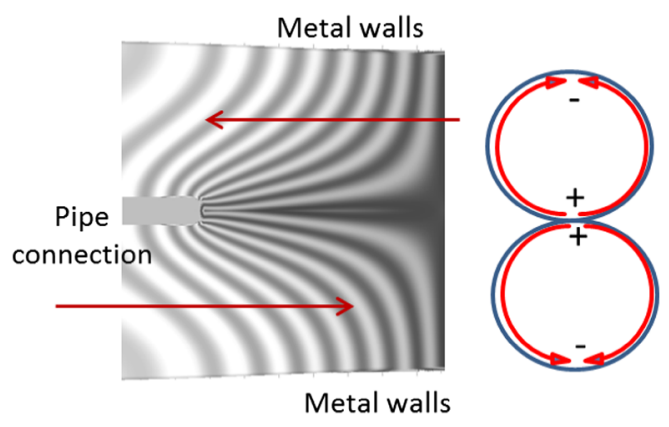

FIG. 3. Electric field line distribution (left plot) and surface current distribution (right plot) of the trapped mode. Each surface current plane has a longitudinal slope of the corresponding sign. The red lines show the direction of the colliding beams.

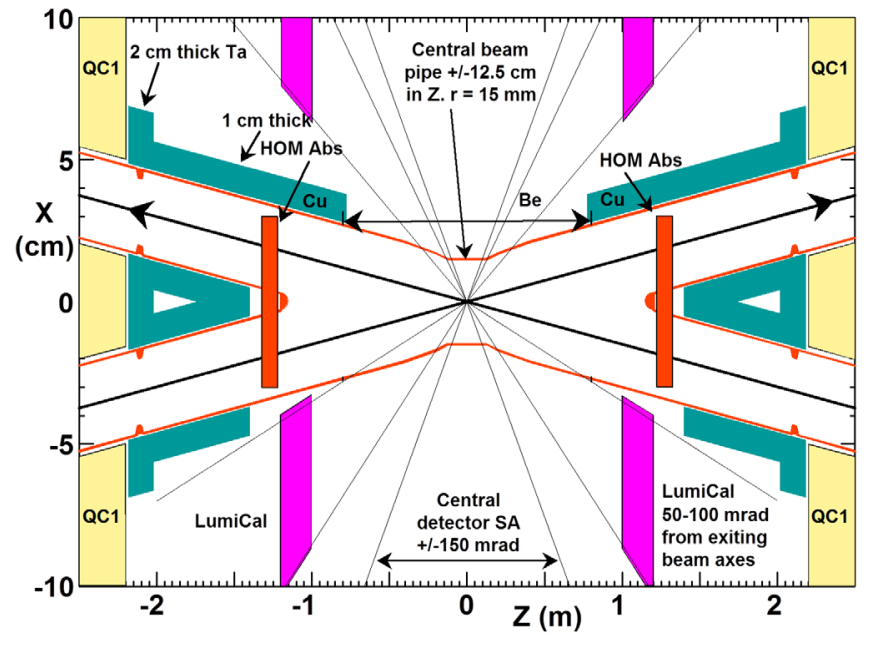

FIG. 4. FCC ee IR. The red lines show the boundary of the IR beam chamber.

connection (identical to first one) due to the opposite sign of the kick.

\section{FCC $\mathrm{E}^{-} \mathrm{E}^{+}$INTERACTION REGION}

A more detailed analysis of the trapped modes was carried out for the intersection region of the FCC ee [16].

The flexible interaction region design for the future electron-positron circular collider $\left(\mathrm{FCC} \mathrm{e}^{-} \mathrm{e}^{+}\right)$allows a large beam energy range as the machine will work at different beam energies, from $45.6 \mathrm{GeV}$ up to $182.5 \mathrm{GeV}$, with a luminosity that goes from $2.3 \times 10^{36} \mathrm{~cm}^{-2} \mathrm{~s}^{-1}$ for the lower energy to $1.5 \times 10^{34} \mathrm{~cm}^{-2} \mathrm{~s}^{-1}$ for the highest one. The circumference of the machine is foreseen to be about $100 \mathrm{~km}$. The crab-waist collision scheme has been chosen to reach the highest possible luminosity, so the crossing angle is $30 \mathrm{mrad}$. For the Z-production, to reach a high luminosity it is planned to have a high current of $1.4 \mathrm{~A}$ in each beam. The bunch length will vary from 2.5 to $12 \mathrm{~mm}$.

All models for the wakefield calculations are based on the Sullivan design, presented at the FCC week 2017 [16]. The sketch of the IR region is shown below in Fig. 4.

The IR has a $2.2 \mathrm{~m} \mathrm{~L}^{*}$ (distance from IP to the face of the first quad QC1) and the final focusing magnets are superconductive. Synchrotron radiation (SR) mask tips are placed at $\pm 2.1 \mathrm{~m}$, in order to protect the central chamber from photons generated by the last soft magnet whose face is located $100 \mathrm{~m}$ from the IP.

\section{THE HOM ANALYSES}

\section{A. Possible smooth pipe connections}

In order to diminish the HOM effects we tried to find a geometry for the IR beam pipe that has the smallest impedance. Among other things we considered three different models of the IR all with the same diameter of the incoming pipes and of a central pipe of $30 \mathrm{~mm}$ in a diameter. 
Model 1.-The incoming pipes are smoothly squeezed to a half circle shape in order to merge into the central pipe with a constant diameter.

Model 2.-The incoming pipes near the connection are circular pipes. The central part near the pipe connection has a transition to an approximately elliptical or racetrack shape with a double size in the horizontal direction. This connection between the two pipes and the elliptical shape contains some small sharp transitions.

Model 3.-Each pipe near the connection has a transition to a half of a special shape determined by the shape of the transition from the central pipe (a proposal from Oide Katsunobu). This is a full smooth geometry.

\section{B. The method of calculations}

The wakefield or HOMs analyses is based on the calculation of the wakefield potential with postprocessing Fourier analyses. We first find the resonances in the spectrum of the wakefield potential. Then we do an eigenmode calculation in the frequency region around this resonance. In this way we know by how much the beam interacts with this mode and the eigenmode calculation gives us the detailed structure information about this mode. We use the CST code for wakefield calculations and we use the CST and HFSS codes for the eigenmode calculations.

We calculate wakefield potentials in a $4 \mathrm{~m}$ long part of the interaction region, which includes all of the important elements. In the calculation we use a Gaussian bunch with $2.5 \mathrm{~mm}$ bunch length, however for an initial quick analysis we did calculations for a 10 and $5 \mathrm{~mm}$ bunch. Initially, we designed the geometry of the IR beam pipes using the CST code editor for the geometry. The first and the second model were done in this way. However for a smooth IR geometry we had a problem with the mesh distribution. The limited possibility of the CST code editor did not allow us to make a smooth geometry and consequently the mesh generator failed to make a good mesh. As this was an important point we decided to use CAD files as input for the CST and HFSS codes. We got CERN support and professional CAD files. This new approach improved the preparation of the files and, what is most important, diminished the problem of the "wild" mesh distribution. The code CATIA [17] was used for preparation of the CAD files. We found the needed file format and additional requirements for the CATIA CAD file to be successfully accepted by the CST code.

\section{Model 1: Squeezed incoming pipes}

The initial calculations were done for the first IR model, which is shown in Fig. 5 [18]. The length of the central beam pipe is $400 \mathrm{~mm}$ and the diameter is $30 \mathrm{~mm}$. The cross section near the pipe connection shows how the incoming pipes were squeezed to the half circle shape.

Results of the wakefield calculations for this geometry are shown in Figs. 6 and 7. The red line in Fig. 6 shows the

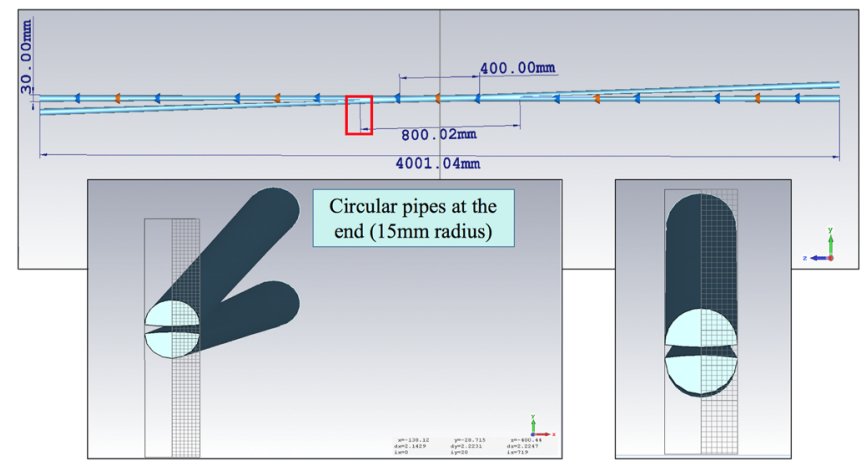

FIG. 5. Geometry of the IR model 1 . The cross section near the pipe connection shows how the incoming pipes were squeezed to the half circle shape.

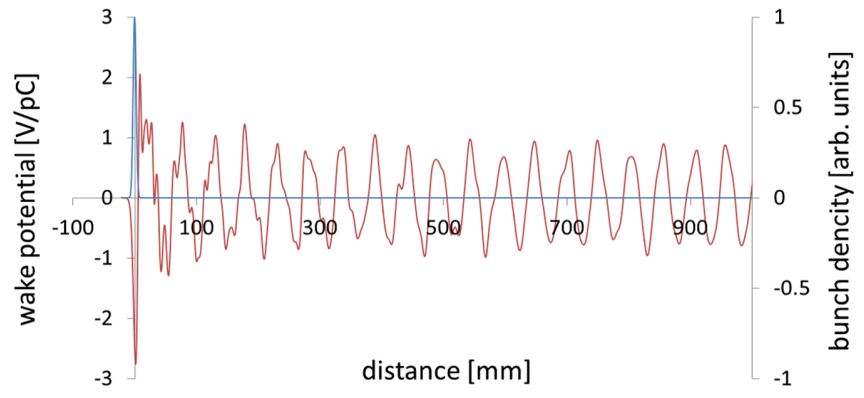

FIG. 6. Wakefield potential (red line) of a $2.5 \mathrm{~mm}$ bunch and a bunch shape (blue line) in the IR model 1.

first $1 \mathrm{~m}$ long part of the calculated wakefield potential for a $2.5 \mathrm{~mm}$ Gaussian bunch. The blue line shows the bunch linear charge density. The total length of the potential is $2 \mathrm{~m}$. We notice a periodic behavior of some part of the wakefield potential. In time this periodic part (a trapped mode) becomes the main part of the potential. Other waves, which have higher frequencies, quickly leave the IR.

We calculate the spectrum of the excited fields using the Fourier transform of the wakefield potential of the total length of $2 \mathrm{~m}$. The real part of the spectrum is shown by the blue line in Fig. 7. The fact that the real part of the spectrum is always positive in the given frequency range demonstrates the high quality of the calculation of the wakefield potential.

The spectrum shows only one trapped mode at the frequency of $5.78 \mathrm{GHz}$. To analyze this mode we performed an eigenmode calculation in the frequency region of 5-7 GHz and found a resonance mode at $5.774 \mathrm{GHz}$. This confirms that the beam excites exactly this mode. The electric field line distribution for this trapped mode is shown in Fig. 8. We see a similar field distribution as the unavoidable mode, which we discussed before (Fig. 3).

\section{Model 2: Small sharp transition}

The geometry of the second IR model is shown in Fig. 11 [18]. In this model the central circular pipe has a transition to an elliptical shape at both sides. The length of each 


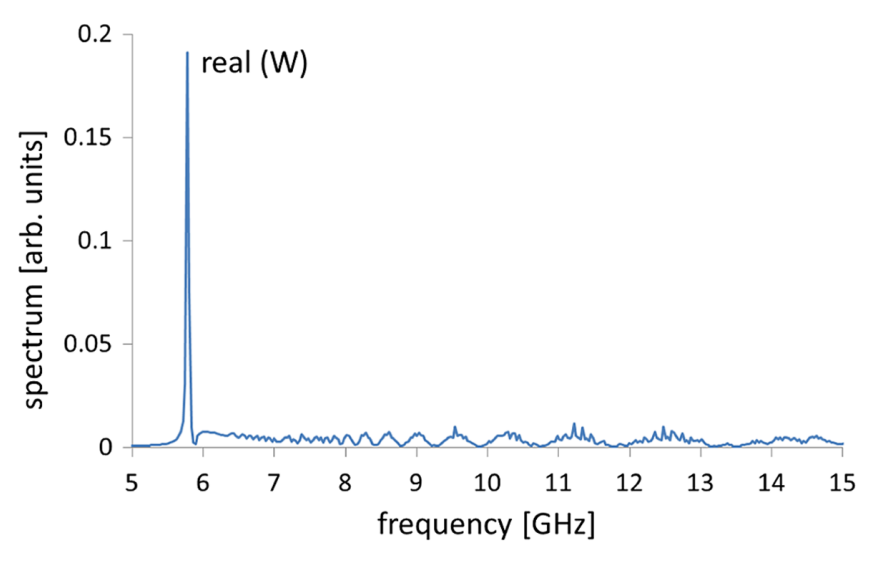

FIG. 7. Spectrum of the excited fields (real part) in the IR model 1.

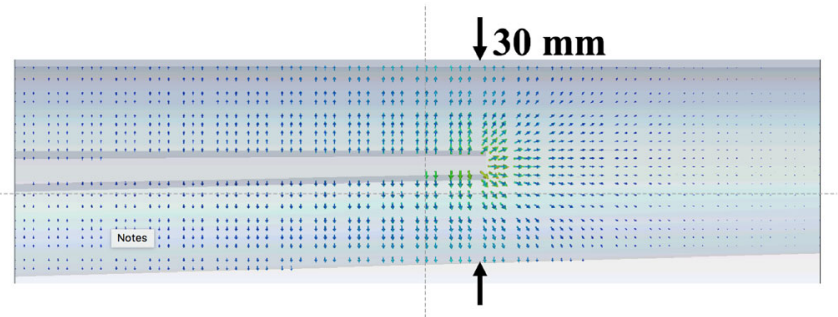

FIG. 8. Electric field line distribution for the trapped mode in model 1.

transition is $0.82 \mathrm{~m}$. We performed wakefield and spectrum calculations and found only one trapped mode at the frequency of $5.67 \mathrm{GHz}$. It is interesting to note that we did see a lower frequency mode (in the region of 3-4 GHz) due to the larger size of the common pipe. Perhaps the sharp transition in this model eliminates this possible low frequency mode. The real part of the spectrum is shown in the lower right plot of Fig. 9. The structure of the distribution of the electric field lines is also similar to the unavoidable mode.

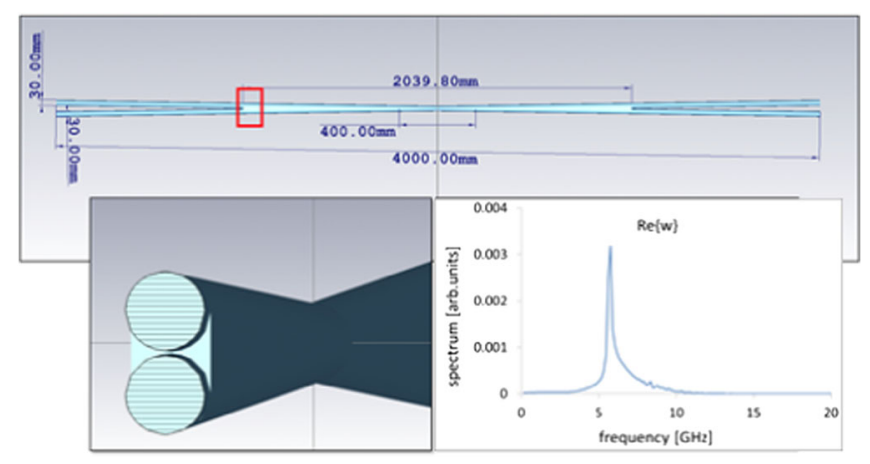

FIG. 9. Geometry of the IR model 2. The cross section near the pipe connection shows two small sharp transitions to the elliptical shape. The spectrum of the exited fields in this model is shown in right lower plot.

\section{E. Model 3: Smooth geometry}

In this model 3 the central circular pipe has two transitions on the left and on the right to the some kind of racetrack shape and each incoming pipe also has a transition to half of this shape, so the two pipes together have a cross section of the connecting central pipe. Details of the transition of two incoming pipes to a central common pipe can be seen in Fig. 10. This is a very smooth geometry. The central inner part of the pipe connection is rounded to make a smooth edge.

The calculated wakefield potential of a $2.5 \mathrm{~mm}$ bunch in the IR model 3 is shown in Fig. 11. Almost all higher frequency modes leave the IR in 2-3 nsec which is just before the arrival of the next bunch. The trapped mode frequency here is almost 2 times lower than the trapped mode frequency in model 1 . The amplitude is 4 times smaller than the amplitude in model 1 (Fig. 6). We may conclude that the impedance of the trapped mode in model 3 is much lower than the impedance of the mode in model 1.

The calculated spectrum (real and imaginary parts) for this model is shown in Fig. 12. The frequency of the trapped mode is $3.46 \mathrm{GHz}$. As we mentioned before, it is lower than the mode found in model 1 due to the larger size of the common pipe. It is interesting to note the broadband

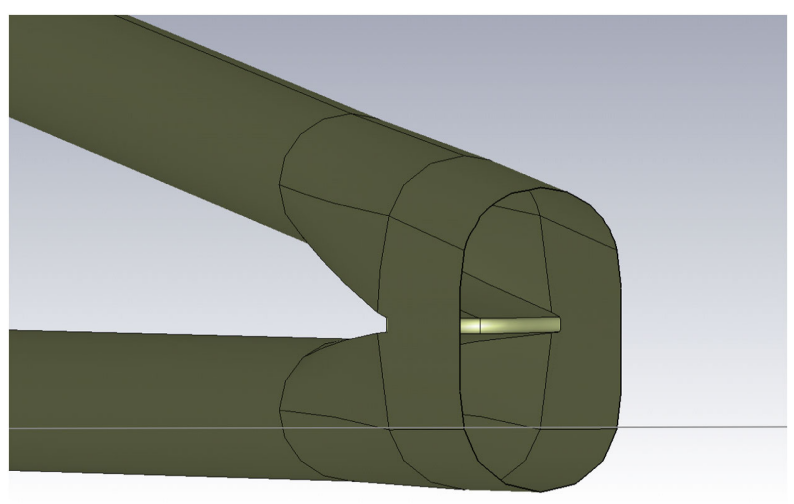

FIG. 10. Inside and outside view of the transition in the IR model 3 .

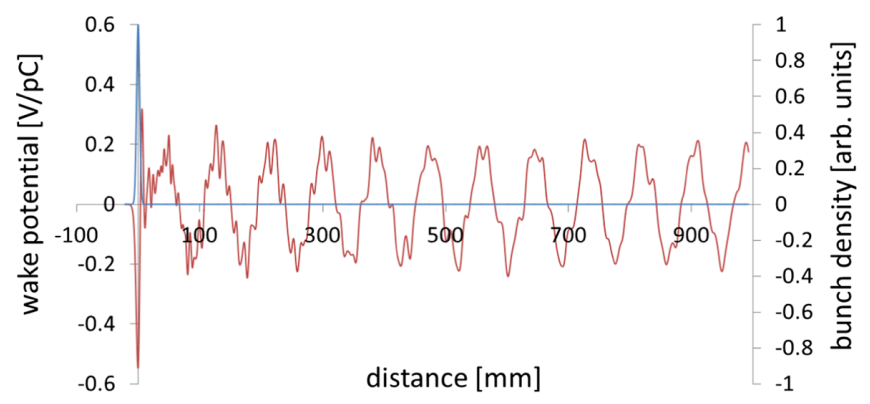

FIG. 11. Wake potential (red line) of a $2.5 \mathrm{~mm}$ bunch in model 3 . The blue line shows bunch linear charge density distribution. 


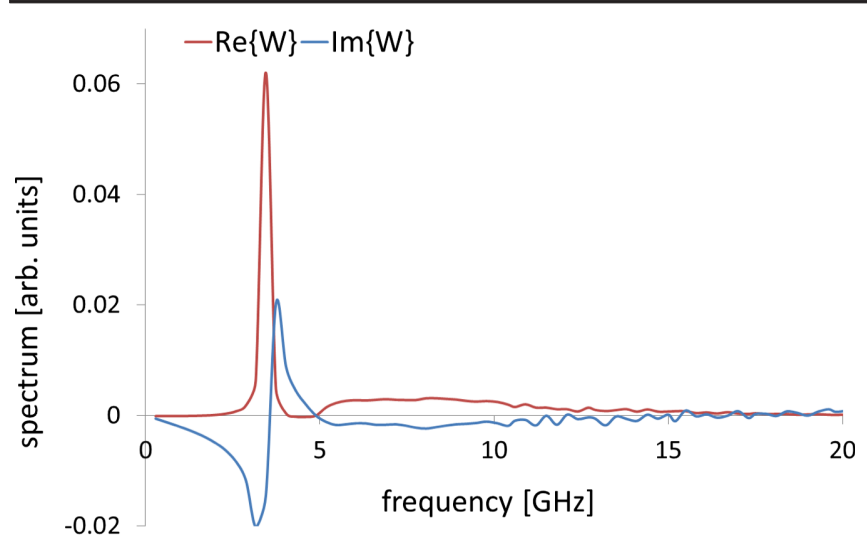

FIG. 12. Real (red line) and imagery (blue line) parts of the wakefield potential spectrum in the IR model 3.

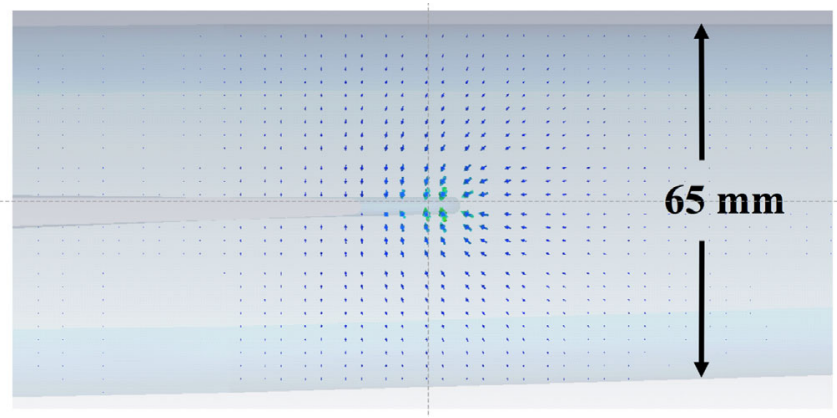

FIG. 13. Electric field line distribution for the trapped mode in model 3.

impedance in the frequency range from 5 to $12 \mathrm{GHz}$. The amplitude is not very high, but perhaps we need more careful calculations for this frequency range.

The electric field line distribution of the trapped mode is shown in Fig. 13. It is almost identical to the trapped mode in model 1 (Fig. 8) and the unavoidable mode in Fig. 3.

\section{HOM ABSORBER CONCEPT}

We now study how to mitigate the effect of the unavoidable mode, which we have found in all three as well as other models. The structure of the mode field distribution shows how we can capture it with a minimum disruption to the image currents of the beam field. The electric field lines in the two incoming pipes are perpendicular to the beam trajectory. We therefore can make longitudinal slots in the top and bottom walls of these pipes (see Fig. 14).

The slots are oriented perpendicular to the HOM electric field. This allows the mode field to easily propagate through these slots. At the same time, the beam field will not pass through these slots. We can then put a watercooled absorber above and below the slots. A sketch of the IR with the geometry of model 3 together with HOMs absorbers is shown in Fig. 15.

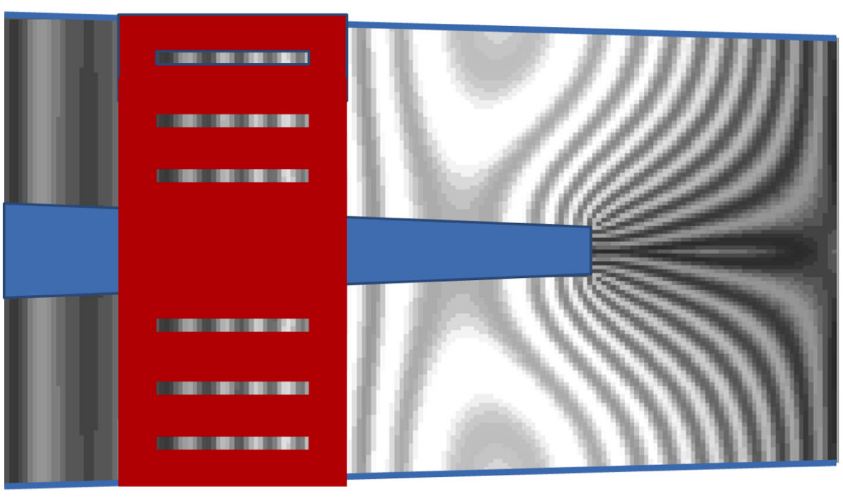

FIG. 14. The geometry (blue lines) of the IR near one of the pipe connections with a fragment of the top wall (red colored plate) with the longitudinal coupling slots. Grey lines show the electric force line of the mode. Electric force lines that can be seen through the slots are perpendicular to the slot. That means that the mode field can escape out through these slots. The slot dimensions and number of them will be optimized for better absorption of the trapped mode.

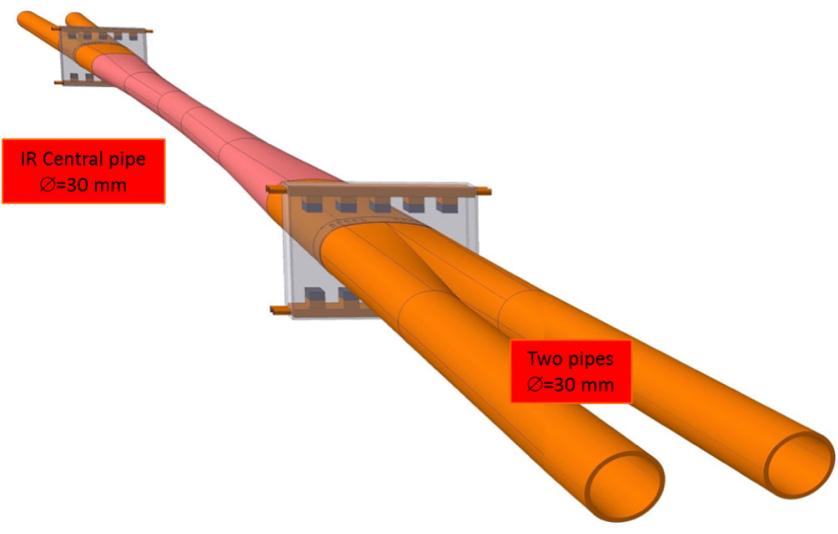

FIG. 15. IR smooth geometry with HOM absorbers.

\section{CALCULATION OF THE HOM HEATING POWER}

To calculate the radiation power we use a cumulative spectral density of the energy losses which is the following integral (details are given in the Appendix):

$$
k(\omega)=\operatorname{Re}\left\{\frac{c}{\pi Q} \int_{0}^{\omega} \rho\left(-\omega^{\prime}\right) W\left(\omega^{\prime}\right) d \omega^{\prime}\right\} .
$$

The plot of this integral for model 3 is shown in Fig. 16.

The spectral density has a sharp step at the resonant frequency of the trapped mode. The size of this step determines the loss factor of the trapped mode. Cutoff frequencies for $\mathrm{TE}_{11}$ and $\mathrm{TM}_{01}$ modes (see the Appendix) are shown by vertical red lines. Above each frequency the transverse fields or longitudinal fields can propagate out of the interaction region. The loss factor of the propagating fields is the part of the density that starts at the cutoff frequency. 


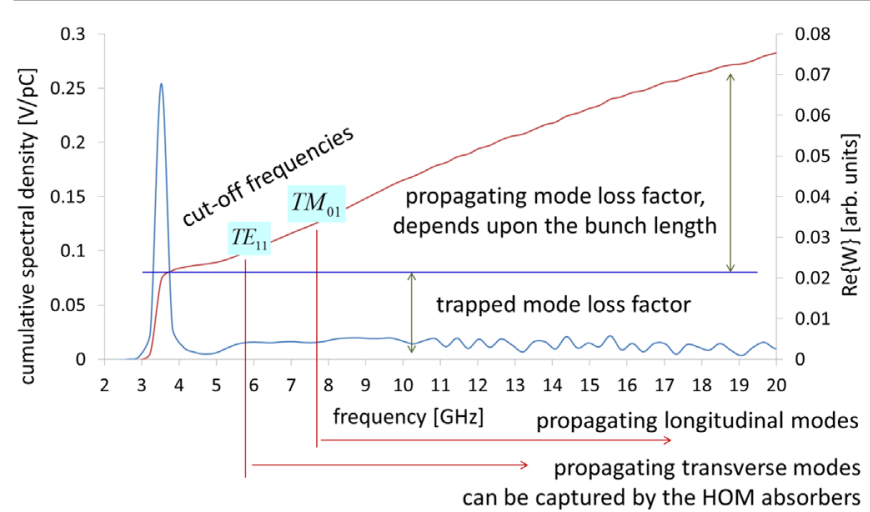

FIG. 16. A cumulative spectral density of the energy losses (red line) and a real part of the spectrum of the excited field (blue line). The horizontal blue line shows the level corresponds to the loss factor of the trapped mode. Above this level we have mainly propagating modes.

Using this plot we can calculate the power loss into the trapped mode using this formula (see the Appendix):

$$
P_{\mathrm{HOM}}=2 I^{2} k_{\mathrm{HOM}} \tau_{l, \mathrm{HOM}},
$$

where $I$ is the beam current, $k_{\mathrm{HOM}}$ is the loss factor of the trapped mode and $\tau_{l, \mathrm{HOM}}$ is the loaded decay time of this mode. The energy loss in the propagating modes is calculated by a similar formula, but now we use a loss factor for the propagating modes $\left(k-k_{\mathrm{HOM}}\right)$ and a bunch spacing $\tau_{b}$,

$$
P_{\text {prop }}=I^{2}\left(k-k_{\mathrm{HOM}}\right) \times \tau_{b} .
$$

We assume that with the water-cooled absorber we can decrease the loaded quality factor of the trapped mode by at least 100. In addition, some fraction of the propagating modes will be captured by the absorber. Estimated numbers for the HOMs heating in the IR with the geometry of model 1 and model 3 and for the beam current of $1.45 \mathrm{~A}$ are shown in Table I. We found later that the mesh in model 2 (which was designed using CST editor) was not very good. Unfortunately we did not repeat calculations using the new CAD approach. For this reason we did not put results for model 2 in the table. We present numbers for the bunch length of 5 and $2.5 \mathrm{~mm}$. The bunch spacing is $2.5 \mathrm{~ns}$. These beam parameters are near the beam parameters of the electron-positron future circular collider, as they are not finally established to this time.

\section{CONCLUSION}

We found that in the interaction region of the colliding beams there is at least one unavoidable trapped mode. We have designed a smooth geometry of the IR vacuum chamber, which has relatively small HOMs impedance and only one trapped mode. This vacuum chamber can be a prototype of the IR chamber of the future circular electronpositron collider. In the case of the bunch length of $2.5 \mathrm{~mm}$ and the beam current of $1.45 \mathrm{~A}$ the electromagnetic power will be approximately $5 \mathrm{KW}$ in each set of pipe connection, which includes power of the trapped mode and the power of all propagating modes. This power will be mainly absorbed in the HOM absorbers. More work will be needed to further optimize the HOM absorbers.

\section{ACKNOWLEDGMENTS}

We would like to thank Frank Zimmermann, Manuela Boscolo and especially Michael Benedikt for their great support of this work. We are also happy to thank Oide Katsunobu, Mauro Migliorati and the MDI team for many useful discussions and help. This work was supported partially by Department of Energy Contract No. DE-AC02$76 \mathrm{SF} 00515$.

\section{APPENDIX: CALCULATION OF THE BEAM ENERGY LOSS DUE TO EXCITATION OF THE HIGHER ORDER MODES}

Many formulas in this chapter can be found elsewhere [19-21], however to clarify all the coefficients we repeat several of them.

\section{Green's function and wakefield potential}

According to the energy conservation law, the power of the excited electromagnetic fields (wakefields) is naturally the power that the beam loses. In order to estimate this power we need to calculate the value of the electromagnetic field that is excited by a charged bunch and that acts back on the single particles of the bunch. Then we can integrate the value of the longitudinal component of this excited electric field $E_{z}(t, z)$ along the particle's trajectory.

\begin{tabular}{|c|c|c|c|c|c|c|c|}
\hline \multirow[b]{2}{*}{$\begin{array}{l}\text { Model } \\
\text { No. }\end{array}$} & \multirow[b]{2}{*}{$\begin{array}{c}\text { Trapped mode } \\
\text { frequency } \\
{[\mathrm{GHz}]}\end{array}$} & \multirow[b]{2}{*}{$\begin{array}{c}\text { Near revolution } \\
\text { harmonic } \\
\text { numbers }\end{array}$} & \multirow[b]{2}{*}{$\begin{array}{l}\text { Mode loss } \\
\text { factor } \\
{[\mathrm{V} / \mathrm{pC}]}\end{array}$} & \multirow[b]{2}{*}{$\begin{array}{c}\text { Mode decay } \\
\text { time } \\
{[\mathrm{ns}]}\end{array}$} & \multirow[b]{2}{*}{$\begin{array}{c}\text { Power of a } \\
\text { trapped mode } \\
{[\mathrm{kW}]}\end{array}$} & \multicolumn{2}{|c|}{ Propagating modes power } \\
\hline & & & & & & $\begin{array}{l}\text { Bunch } \\
5 \mathrm{~mm} \\
{[\mathrm{~kW}]}\end{array}$ & $\begin{array}{c}\text { Bunch } \\
2.5 \mathrm{~mm} \\
{[\mathrm{~kW}]}\end{array}$ \\
\hline I & 5.774 & 14 and 15 & 0.38 & 5.51 & 8.71 & 2.42 & 10.77 \\
\hline III & 3.459 & 8 and 9 & 0.08 & 9.2 & 2.91 & 0.45 & 2.10 \\
\hline
\end{tabular}

TABLE I. RF parameters of a trapped mode in the model 1 and model 3. 
This integral which depends upon the longitudinal particle position in the bunch $s$, is called a wakefield potential $W(s)$ :

$$
W(s)=\frac{1}{Q} \int_{-\infty}^{\infty} E_{z}(t, z)_{z=c t-s} c d t
$$

The wake potential is usually normalized to the total bunch charge $Q$ and measured in $\mathrm{V} / \mathrm{pC}$. Wakefields and correspondent wake potentials can be calculated by integrating Maxwell's equations in the time domain using numerical codes [3-5].

A wakefield potential of a point charge can serve as a Green's function $G(s)$ and can be used for calculation of the wakefield potentials of the bunches of any line charge density distribution $\rho(s)$ :

$W(s)=\frac{1}{Q} \int_{-\infty}^{s} \rho\left(s^{\prime}\right) G\left(s-s^{\prime}\right) d s^{\prime}=\frac{1}{Q} \int_{0}^{\infty} \rho\left(s-s^{\prime}\right) G(s) d s^{\prime}$.

A loss factor $k$, which determines the bunch energy loss, is calculated as an integral of the convolution of the wake potential and the bunch charge density distribution,

$$
k=\frac{1}{Q} \int_{-\infty}^{\infty} W(s) \rho(s) d s
$$

It has the same dimensions as a wakefield potential; however the total bunch energy loss $\Delta \mathcal{E}$ is determined by the loss factor, which is multiplied by the total bunch charge squared,

$$
\Delta E=k Q^{2} .
$$

\section{Frequency spectrum of the wakefield potential and cumulative spectral density of energy losses}

Here and later we assume that the particles of the bunch have ultrarelativistic velocities $v \cong c$, where $c$ is the speed of light. The Fourier transform of the wakefield potential $W(\omega)$ is defined as an integral,

$$
W(\omega)=\int_{-\infty}^{\infty} W(s=c \tau) \exp (-i \omega \tau) d \tau
$$

As the wakefield potential is a real function then the real part of the transform is a symmetric function of the frequency while the imaginary part is an asymmetrical function of frequency [19].
Using the inverse Fourier transform

$$
W(t)=\frac{1}{2 \pi} \int_{-\infty}^{\infty} W(\omega) \exp (i \omega \tau) d \omega,
$$

we can present a loss factor in the following way:

$$
\begin{aligned}
k & =\frac{1}{Q} \int_{-\infty}^{\infty}\left[\rho(s=c t)\left(\frac{1}{2 \pi} \int_{-\infty}^{\infty} W(\omega) \exp (i \omega t) d \omega\right)\right] c d t \\
& =\frac{c}{2 \pi Q} \int_{-\infty}^{\infty} \rho(-\omega) W(\omega) d \omega
\end{aligned}
$$

where $\rho(\omega)$ is a Fourier transform of the bunch line charge density distribution,

$$
\rho(\omega)=\int_{-\infty}^{\infty} \rho(s=c \tau) \exp (-i \omega \tau) d \tau .
$$

For a single bunch that has a Gaussian shape for the charge density distribution with the rms bunch length $\sigma$ and the total charge $Q_{b}$ the Fourier transform is

$$
\rho(\omega)=\frac{1}{c} Q_{b} e^{-\frac{1}{2}\left(\omega \frac{\sigma}{c}\right)^{2}} .
$$

If the spectrum of the bunch density distribution has only a real part (like a Gaussian distribution) then we need only the real part of the spectrum of the wakefield potential.

Based on (A7) and changing the upper limit of the integral to a finite value, we will introduce a cumulative spectral density of the energy losses,

$$
k(\omega)=\operatorname{Re}\left\{\frac{c}{\pi Q} \int_{0}^{\omega} \rho\left(-\omega^{\prime}\right) W\left(\omega^{\prime}\right) d \omega^{\prime}\right\} .
$$

The cumulative spectral density of the energy losses is shown in Fig. 5. We can see a sharp step near the resonance. We can calculate the intrinsic impedance $R / Q$ of this resonance using the following formula, where $\Delta k$ is the size of this step (loss factor of this mode):

$$
R / Q=2 \frac{\Delta k}{\omega} .
$$

The frequency spectrum of the wakefield potential shows us if any resonance exists in the low frequency range. It may reflect the fact that low frequency modes may be trapped in some kind of cavity and oscillate there for some time. Actually this time is determined by the loaded quality factor of this mode. These electromagnetic fields 
after a time of several oscillations will leak out of the cavity and propagate down the beam pipe. For this reason in the high frequency range the spectrum is a more or less smooth function of the frequency. We may assume that the frequency which separates different spectrum behavior is the cutoff frequency.

\section{Cutoff frequencies}

Usually we consider some geometry (like a cavity), which is different to the geometry of the incoming and outgoing pipes. In our case this is the connection of two beam pipes. The cutoff frequency is the minimum frequency of the electromagnetic wave, which may propagate in the incoming and outgoing pipes. Below this frequency the field can be trapped in the "cavity". The cutoff frequency is determined by the geometry of the beam pipe. There can be several types of propagating waves like transverse magnetic (TM) or transverse electric (TE). For a round beam pipe of radius $a$ the minimum cutoff frequency is the frequency of the longitudinal TM01 mode,

$$
f_{\mathrm{TM}_{01}}^{\text {cutoff }}=\frac{c}{a} \times \frac{\nu_{01}}{2 \pi} ; \quad \nu_{01}=2.4048 .
$$

In our calculations the radius of the beam pipe is $15 \mathrm{~mm}$ that corresponds to a cutoff frequency of $7.65 \mathrm{GHz}$. However a transverse mode TE11 has a lower cutoff frequency:

$$
f_{\mathrm{TE}_{11}}^{\text {cutoff }}=\frac{c}{a} \times \frac{\mu_{11}}{2 \pi} ; \quad \mu_{11}=1.8412 .
$$

In our case it is $5.86 \mathrm{GHz}$. This mode has a transverse electric component and may propagate through the longitudinal slots, or between the fingers of shielded elements like bellows and valves. A plot of the cumulative spectral density of energy loss (Fig. 19) will give us information on how much energy a bunch loses for modes below or above the cutoff frequency.

\section{Power loss of a train of bunches below and above cutoff frequency}

According to [20] the beam power loss due to the resonant excitation of a trapped mode is

$$
\begin{aligned}
P_{n} & =I^{2}\left(\frac{R}{Q}\right)_{n} Q_{l} f\left(\tau_{l}, \tau_{b}\right) \\
f\left(\tau_{l}, \tau_{b}\right) & =\frac{\frac{\tau_{b}}{\tau_{l}}\left[1-\exp \left(-2 \frac{\tau_{b}}{\tau_{l}}\right)\right]}{2\left[1-2 \exp \left(-\frac{\tau_{b}}{\tau_{l}}\right) \cos (\Delta \omega) \tau_{b}+\exp \left(-2 \frac{\tau_{b}}{\tau_{l}}\right)\right]} .
\end{aligned}
$$

In the case where the decay time of this mode $\tau_{l}=\frac{2 Q_{l}}{\omega}$ is much longer than the bunch spacing $\tau_{b}$, we can write the following:

$$
P_{n}=2 k_{n} I^{2} \tau_{l}
$$

In the opposite case where the mode decays before the arrival of the next bunch, the power loss is

$$
P_{n}=k_{n} I^{2} \tau_{b}
$$

[1] A. Novokhatski, J. Seeman, and M. Sullivan, Analysis of the wakefield effects in the PEP-II storage rings with extremely high currents, Nucl. Instrum. Methods Phys. Res., Sect. A 735, 349 (2014).

[2] A. Novokhatski, J. Seeman, M. Sullivan, and U. Wienands, Electromagnetic waves excitation, propagation and absorption in the high current storage rings, IEEE Trans. Nucl. Sci. 63, 812 (2016).

[3] A. Novokhatski, Field dynamics of coherent synchrotron radiation using a direct numerical solution of Maxwell's equations, Phys. Rev. ST Accel. Beams 14, 060707 (2011).

[4] User Guide, CST GmbH, Darmstadt, Germany, https:// www.cst.com/.

[5] HFSS, http://www.ansys.com/products/electronics/ansyshfss.

[6] S. Weathersby and A. Novokhatski, Wakefields in the Super B factory interaction region, in Proceedings of the International Particle Accelerator Conference, Kyoto, Japan (ICR, Kyoto, 2010), TUPD026, pp. 2105-2107.

[7] A. Novokhatski and M. Sullivan, Beam fields and energy dissipation inside the Be beam pipe of the Super-B detector, in Proceedings of the International Particle Accelerator Conference, Kyoto, Japan (Ref. [6]), TUPEB081, pp. 15781580.

[8] K. Shibata and K. Kanazawa, Loss factor and impedance of IR beam duct for Super-KEKB and KEKB, in Proceedings of the International Particle Accelerator Conference, Kyoto, Japan (Ref. [6]), TUPD042.

[9] R. Wanzenberg et al., Calculations of wakefields for the new design of the LHCb vertex locator, CERN, Geneva, Switzerland, Report No. CERN-ACC-NOTE-2017-0034, 2017.

[10] R. Wanzenberg et al., Calculation of wakefields and higher order modes for the vacuum chamber of the ATLAS experiment for the HL-LHC, CERN, Geneva, Switzerland, Report No. CERN-ACC-NOTE-2013-0046, 2013.

[11] A. Novokhatski, Wake potentials in the ILC interaction region, in Proceedings of the 24th Particle Accelerator Conference, PAC-2011, New York, 2011 (IEEE, New York, 2011), pp. 1837-1839.

[12] G. Stupakov, Estimates of local heating due to trapped modes in vacuum chamber, MDI Meeting, CERN, Geneva, Switzerland, 2016.

[13] A. Novokhatski, Overall HOM measurement at the high beam currents in the PEP-II SLAC B-factory, in Proceedings of the 22nd Particle Accelerator Conference, PAC2007, Albuquerque, NM (IEEE, New York, 2007) MOOAKI02, pp. 45-47.

[14] A. Novokhatski, S. DeBarger, S. Ecklund, N. Kurita, J. Seeman, M. Sullivan, S. Weathersby, and U. Wienands, 
A new Q-2 bellows absorber for the PEP-II SLAC B-factory, in Proceedings of the 22nd Particle Accelerator Conference, PAC-2007, Albuquerque, NM (Ref. [13]), FRPMS076, pp. 4219-4221.

[15] M. Benedikt and F. Zimmermann, Towards future circular colliders, J. Korean Phys. Soc. 69, 893 (2016).

[16] FCC week 2017, Berlin, Germany, https://indico.cern.ch/ event/556692/timetable/\#20170531.detailed.

[17] CATIA, http://www.catia.com.pl/.

[18] E. Belli, Trapped mode analysis, FCC-ee MDI workshop, 2017, CERN, Geneve, Switzerland, https://indico.cern.ch/ event $/ 596695 /$.
[19] B. Zotter and S. Kheifets, Impedances and Wakes in High Energy Particle Accelerators (World Scientific Publishers, Singapore, 1998).

[20] L. Merminga et al., Specifying HOM-power extraction efficiency in a high average current, short bunch length SRF environment, in Proceedings of the 20th International Linac Conference, LINAC-2000, Monterey, CA, 2000 (SLAC, Menlo Park, CA, 2000), p. 860.

[21] A. Novokhatski, HOM calculations of new rf cavities for super B-factory, in Proceedings of the Super B Factory Workshop in Hawaii (Report No. SLAC-PUB-10792, 2004). 